\title{
THE INTERVAL OF DISK PACKING EXPONENTS ${ }^{1}$
}

\author{
J. B. WILKER
}

Abstract. The set of disk packing exponents is an interval equal to $(E, 2]$ or $[E, 2]$. The set of triangle packing exponents is $\left[\log _{2} 3,2\right]$. The analogy strongly suggests that $E$ is attained and that $E=S$, the osculatory exponent whose value is known to lie in the interval $1.0300197<S<1.314534$.

1. Literature survey and statement of theorems. Let $U$ denote a disk of diameter 1 in the Euclidean plane. A countable collection of open disks $C=\left\{D_{m}\right\}, D_{m}$ of diameter $d_{m}$, is called a packing of $U$ in case its members are contained in $U$ and disjoint from one another. If the residual set of uncovered points $R(C)=U-\bigcup_{m=1}^{\infty} D_{m}$ has plane Lebesgue measure 0 then $C$ is called an almost perfect packing (alternatively a solid or complete packing). As this paper deals exclusively with almost perfect packings it will not be ambiguous to refer to them simply as packings.

The fact that packings actually exist may be proved by appealing to the Vitali covering theorem or by constructing an explicit example (cf. Kasner and Supnick [12]). Let me outline progress in two related areas of packing theory-study of the asymptotic behavior of diameter sequences $d_{m}$ and study of the Hausdorff dimension of residual sets $R(C)$. References to possible physical applications of packing theory are provided by Gilbert [10] while Davis [7] shows how packings may be employed to give quadrature formulae.

Interest in the packing diameters was sparked by Mergelyan's observation $[17, \S 4]$ that, while $\sum_{m=1}^{\infty} d_{m}^{2}=1$, the series $\sum_{m=1}^{\infty} d_{m}$ must diverge. Wesler [19] gave a simple proof of this theorem and Melzak [15] introduced the moment sum of a packing,

$$
M(C, \alpha)=\sum_{m=1}^{\infty} d_{m}^{\alpha} \quad(\alpha \text { real }),
$$

and defined the exponent of a packing,

$$
e(C)=\inf \{\alpha: M(C, \alpha)<\infty\} .
$$

Received by the editors January 24, 1973.

AMS (MOS) subject classifications (1970). Primary 52A45; Secondary 28A75.

Key words and phrases. Almost perfect packing, osculatory packing, disk packing exponent, Hausdorff dimension.

${ }^{1}$ Supported in part by Canadian N.R.C. grant A8100. 
As the preceding remarks indicate, packing exponents must satisfy $1 \leqq$ $e(C) \leqq 2$. Melzak constructed one packing with exponent 2 and another, which he called an osculatory packing, with exponent $S \neq 2$ and satisfying $1.035<S<1.999971$. Later [16] he gave the heuristic estimate $S \simeq 1.306951$.

To obtain an osculatory packing of $U$, insert two disks of diameter $\frac{1}{2}$ tangent to one another at the centre of $U$. Then proceed by induction inserting at each stage the largest disks that will fit into the set of curvilinear triangles which comprise the current residual. Since these disks are a solution to the Apollonian tangency problem for their three ancestral neighbours, this packing has also been called an Apollonian packing. However, I prefer the allusion to Soddy's Kiss Precise [18], a poem describing the size of such disks.

Many of the other papers which deal with the asymptotics of the sequence of packing diameters ([1], [4], [5], [6], [9], [20]) have enlarged the class of packings with exponent $S$ and gradually improved the bounds on $S$. The best result to date is Boyd's $1.300197<S<1.314534$ [6]. The tools used in these investigations have included elementary geometry, conformal mapping, the analytic theory of matrices, real inequalities and a number of ad hoc arguments.

There has also been interest in determining precisely which numbers can be packing exponents. In [21, $\S \S 5.2$ and 5.3] there is a rather long argument which proves, among other results, that every number in the closed interval $[S, 2]$ is an exponent. In [3] there is a shorter proof of the stronger result that if $B$ is the infimum of the analogously defined exponents for packing the region bounded by two externally tangent circles and a common tangent line by disks then every number in the interval $(B, 2]$ (or $[B, 2]$ if $B$ is attained) is the exponent of some disk packing of $U$. The main purpose of this note is to give a very short proof of the still stronger result,

THEOREM 1. Let $E$ be the infimum of the set of exponents for disk packings of the disk $U$. Then for every number e satisfying $E<e \leqq 2$ there is a packing $C_{e}$ with exponent $e$ and $M\left(C_{e}, e\right)<\infty$. For every number $e$ satisfying $E<e<2$ there is a packing $C_{e}^{\infty}$ with exponent e and $M\left(C_{e}^{\infty}, e\right)=\infty$.

It is quite reasonable to expect that the infimum $E$ is attained and that $E=S$ so that the set of disk packing exponents is the closed interval $[S, 2]$. A second purpose of this paper is to lend support to this conjecture by describing the analogously defined exponents for packing a unit equilateral triangle $T$ by oppositely oriented equilateral triangles. While earlier results along the lines of Theorem 1 were quite specific to disk packings, the present technique has the advantage of being immediately 
available for all packing problems in which a set is packed by homothetic sets. In particular a slight modification of the method gives much of

THEOREM 2. For the case of an equilateral triangle $T$ packed by oppositely oriented equilateral triangles the set of exponents is the closed interval $\left[\log _{2} 3,2\right]$. Packings with exponent 2 satisfy $M(C, 2)=1$ while packings with exponent $\log _{2} 3$ satisfy $M\left(C, \log _{2} 3\right)=\infty$. For all other exponents $e$ it is possible to find packings $C_{e}$ and $C_{e}^{\infty}$ with exponent $e$ such that $M\left(C_{e}, e\right)<\infty$ and $M\left(C_{e}^{\infty}, e\right)=\infty$.

To obtain a triangle packing which realizes the minimum exponent, insert the unique packing triangle of diameter $\frac{1}{2}$ and note that it breaks the unpacked residual into 3 triangles similar to the original. Then proceed by induction inserting at the $n$th stage the $3^{n}$ triangles of maximal diameter $\left(\frac{1}{2}\right)^{n+1}$ that will fit into the set of congruent triangles which comprise the current residual. It is obvious that this process generates a packing with exponent $\log _{2} 3 \simeq 1.58$ and that the osculatory disk packing with exponent $S \simeq 1.30$ is a close relative. The proof that $\log _{2} 3$ is actually the minimum triangle packing exponent follows from a theorem concerning exponents and residual set dimension.

In [8] Eggleston proves that if $C$ is a packing of $T$ by oppositely oriented equilateral triangles then $\operatorname{dim} R(C) \geqq \log _{2} 3$ and the Hausdorff measure of the residual set in this dimension satisfies $m^{\log _{2} 3}(R(C)) \geqq 1 / 3$. In [14] Larman proves that if $C$ is a packing of the $n$-dimensional hypercube by balls then $e(C) \geqq \operatorname{dim} R(C)$. Boyd's related investigations [2] bring out the intuitive reason why an inequality of this sort might be expected. In $[21, \S 2.3]$ the setting of this inequality is generalized to include, among others, the packings of $U$ and $T$ considered here. Moreover, for these packings and others it is shown that $M(C, e(C))<\infty$ implies $m^{e(C)}(R(C))=$ 0 . Thus if $C$ is a packing of $T$ by oppositely oriented equilateral triangles $e(C) \geqq \operatorname{dim}(R(C)) \geqq \log _{2} 3$. Moreover, if $C$ has exponent $\log _{2} 3$, $m^{e(C)}(R(C)) \geqq 1 / 3$ and so $M(C, e(C))=\infty$.

The exponent theory for disk packings of $U$ could be completed by an appropriate analog of Eggleston's theorem. However, there are only sparse results in this difficult area. Hirst [11] has shown that for disk packings of the unit square analogous to the osculatory packing of $U$

$$
1.001<\operatorname{dim}(R(C)) \leqq \log _{(1+2 / \sqrt{ } 3)} 3 \simeq 1.43113 .
$$

Larman [13] has improved the lower bound and generalized the results to show that for arbitrary disk packings of the unit square $\operatorname{dim} R(C)>1.03$.

2. Proof of the theorems. A few preliminary remarks are in order. If $C$ is a packing of $U$ then $M(C, \alpha)=\sum_{m=1}^{\infty} d_{m}^{\alpha}$ is strictly decreasing for 
$\alpha>e(C)$. For $\lambda>0$ we denote by $\lambda C$ the packing of a disk of diameter $\lambda$ obtained by dilating $C$ and therefore

$$
M(\lambda C, \alpha)=\sum_{m=1}^{\infty}\left(\lambda d_{m}\right)^{\alpha}=\lambda^{\alpha} \sum_{m=1}^{m} d_{m}^{\alpha}=\lambda^{\alpha} M(C, \alpha) .
$$

In particular we may take a disk $D_{m}^{\prime}$ out of a packing $C^{\prime}$ and replace it by $d_{m}^{\prime} C$. This is called iterating $C$ over $D_{m}^{\prime}$ of $C^{\prime}$. If we simultaneously iterate $C$ over each of the disks of $C^{\prime}$ then the product packing $C^{\prime} C$ satisfies

$$
M\left(C^{\prime} C, \alpha\right)=\sum_{m=1}^{\infty} M\left(d_{m}^{\prime} C, \alpha\right)=\sum_{m=1}^{\alpha} d_{m}^{\prime \alpha} M(C, \alpha)=M\left(C^{\prime} \alpha\right) M(C, \alpha) .
$$

The fact that iterates and products are again packings follows because measure zero is preserved by dilatation and countable union. These two constructions were first used by Melzak in [15].

To prove Theorem 1 let $e$ satisfy $E<e<2$ and choose $C$ with $e(C)<e$. Write $M(C, \alpha)=\mu(\alpha)$ and note that, for $e(C)<\alpha<2, \mu(\alpha)>\mu(2)=1$. Form $C^{n}$ recalling $M\left(C^{n}, \alpha\right)=\mu(\alpha)^{n}$. Partition the interval $0<x \leqq 1$ by the intervals

$$
I_{n}=\left\{x: \mu(e)^{-n}<x \leqq \mu(e)^{1 \cdot n}\right\} \quad(n=1,2, \cdots) .
$$

Choose subsequences of disks $D_{m(i)}$ in $C$ and intervals $I_{n(i)}$ in the partition such that $d_{m(i)}^{e}$ belongs to $I_{n(i)}$. Iterate $C^{n(i)}$ over $D_{m(i)}(i=1,2, \cdots)$ to get $C_{e}^{\infty}$. Clearly the convergence of $M\left(C_{e}^{\infty}, \alpha\right)$ depends on

$$
\sum_{i=1}^{\infty} M\left(d_{m(i)} C^{n(i)}, \alpha\right)=\sum_{i=1}^{\infty} d_{m(i)}^{\alpha} \mu(\alpha)^{n(i)} .
$$

At $\alpha=e, d_{m(i)}^{e}>\mu(e)^{-n(i)}$, the series is bounded below by $\sum_{i \rightarrow 1}^{\infty} 1$ and $M\left(C_{e}^{\infty}, e\right)=\infty$. However for $\alpha>e, d_{m(i)}^{\alpha}<\mu(e)^{1 \cdot n(i)}$ and the series is bounded above by

$$
\mu(e) \sum_{i=1}^{\infty}\left|\frac{\mu(\alpha)}{\mu(e)}\right|^{n(i)}<\mu(e) \sum_{n-1}^{\infty}\left|\frac{\mu(\alpha)}{\mu(e)}\right|^{n}
$$

which is a convergent geometric progression because $\mu(\alpha)$ is strictly decreasing. This shows that $e\left(C_{e}^{\infty}\right)=e$.

To complete the proof of Theorem 1 it is necessary to produce, for each $e$ satisfying $E<e \leqq 2$, a packing $C_{e}$ with exponent $e$ and moment sum $M\left(C_{e}, e\right)<\infty$. Let $C_{n}(n=0,1, \cdots)$ be a sequence of packings such that the corresponding sequence of exponents $e\left(C_{n}\right)=e_{n}$ is strictly increasing with limit $e$. Since $e_{n}<e, M\left(C_{n}, e\right)$ is finite and there exists a disk $D_{m(n)}$ of $C_{0}$ such that $d_{m(n)}^{e} M\left(C_{n}, e\right)<2^{n}(n=1,2, \cdots)$. Let $C_{e}$ be 
the packing obtained by iterating $C_{n}$ over $D_{m(n)}(n=1,2, \cdots)$. Then $M\left(C_{e}, e\right)<M\left(C_{0}, e\right)+1<\infty$. However, if $\alpha<e$ then $\alpha<e_{n}$ for some $n$, $M\left(C_{e}, \alpha\right)$ diverges with $M\left(C_{n}, \alpha\right)$ and we obtain $e\left(C_{e}\right)=e$.

Theorem 2 deals with packings of $T$ by oppositely oriented equilateral triangles. The remarks of $\S 1$ have established that the infimum of their exponents is $\log _{2} 3$, that the infimum is attained and that $M(C, e(C))=\infty$ for every packing $C$ with $e(C)=\log _{2} 3$. What remains to be proved is the exact analogue of Theorem 1 . The preceding proof would apply immediately except for one complication. The iterate of a packing over a triangle in another packing yields triangles oriented like $T$ rather than oppositely. The simple remedy is to always iterate even powers of packings. In the construction of $C_{e}^{\infty}$ this can be accomplished by iterating $C^{n(i)+1}$ over $D_{m(i)}$ when $n(i)$ is odd. In the construction of $C_{e}$, it suffices to iterate $C_{n}^{2}$ over triangles $D_{m(n)}$ of $C_{0}$ chosen so that $d_{m(n)}^{e}\left[M\left(C_{n}, e\right)\right]^{2}<2^{-n}$.

\section{REFERENCES}

1. D. W. Boyd, Lower hounds for the disk-packing constant, Math. Comp. 24 (1970), 697-704. MR 43 \#3924.

2. - Osculatory packings by spheres, Canad. Math. Bull. 13 (1970), 59-64. MR 41 \#4387.

3. - Disk packings which have non-extreme exponents, Canad. Math. Bull. 15 (1972), 341-344.

4. -_, On the exponent of an osculatory packing, Canad. J. Math. 23 (1971), 355 363. MR $42 \# 6723$.

5. ——, The disk-packing constant, Aequationes Math. 7 (1971), 182-193.

6. - Improved bounds for the disk-packing constant, Aequationes Math. (to appear).

7. P. J. Davis, Simple quadratures in the complex plane, Pacific J. Math. 15 (1965), 813-824. MR 32 \#6114.

8. H. G. Eggleston, On closest packing by equilateral triangles, Proc. Cambridge Philos. Soc. 49 (1953), 26-30. MR 14, 679.

9. D. M. E. Foster, On the exponent of the Apollonian packing of circles, J. London Math. Soc. (2) 3 (1971), 281-287. MR 43 \#2612.

10. E. N. Gilbert, Randomly packed and solidly packed spheres, Canad. J. Math. 16 (1964), 286-298. MR 28 \#5382.

11. K. E. Hirst, The Apollonian packing of circles, J. London Math. Soc. 42 (1967), 281-291. MR 35 \#876.

12. E. Kasner and F. Supnick, The Apollonian packing of circles, Proc. Nat. Acad. Sci. U.S.A. 29 (1943), 378-384. MR 5, 106.

13. D. G. Larman, On the Besicovitch dimension of the residual set of arbitrary packed disks in the plane, J. London Math. Soc. 42 (1967), 292-302. MR 35 \#877.

14. - On the exponent of convergence of a packing of spheres, Mathematica 13 (1966), 57-59. MR 34 \#1928.

15. Z. A. Melzak, Infinite packings of disks, Canad. J. Math. 18 (1966), 838-852. MR 34 \#3443.

16. - On the solid packing constant for circles, Math. Comp. 23 (1969), 169172. MR 39 \#6179. 
17. S. N. Mergelian, Uniform approximations to functions of a complex variable, Uspehi Mat. Nauk 7 (1952), no. 2 (48), 31-122; English transl., Amer. Math. Soc. Transl. (1) 3 (1962), 294-391. MR 14, 547.

18. F. Soddy, The kiss precise, Nature 137 (1936), 1021.

19. O. Wesler, An infinite packing theorem for spheres, Proc. Amer. Math. Soc. 11 (1960), 324-326. MR 22 \#2936.

20. J. B. Wilker, Open disk packings of a disk, Canad. Math. Bull. 10 (1967), 395415. MR 35 \#6041.

21. —_, Almost perfect packings, Thesis, University of Toronto, 1968.

Department of Mathematics, University of Toronto, Toronto, Ontario, CANADA 